

\title{
Vigilancia activa de convulsiones febriles vinculadas a vacuna pentavalente en un hospital centinela en Uruguay
}

\author{
Carlos Zunino1, Germán Botto², Noelia Speranza³, Beatriz Gamio', Karina Malan', Rafael Alonso², \\ Salomé Fernández ${ }^{4}$, Adriana Varela ${ }^{3}$, Teresa Picón ${ }^{3}$ y Gustavo Giachetto ${ }^{1}$
}

\footnotetext{
'Departamento de Pediatría, Facultad de Medicina, UdelaR. ${ }^{2}$ Departamento Métodos Cuantitativos, Facultad de Medicina UdelaR.

${ }^{3}$ Unidad de Inmunizaciones, División Epidemiología, Ministerio

Salud.

${ }^{4}$ Unidad de Farmacovigilancia, División Evaluación Sanitaria Ministerio de Salud.

Los autores no presentan conflicto de interés.

Los autores no cuentan con financiamiento externo.

Recibido: 5 de mayo de 2019 Aceptado: 10 de octubre de 2019

Correspondencia a: Carlos Zunino careduzunino@gmail.com
}

\section{Introducción}

L as vacunas son medicamentos biológicos diseñados con el fin de prevenir la aparición de enfermedades, con alto impacto en la morbimortalidad de la población. Constituyen uno de los mayores logros de la salud pública en la prevención de las enfermedades infecciosas ${ }^{1}$. Si bien tienen buen perfil de seguridad, es importante vigilar la aparición de eventos adversos vinculados a su uso, sobre todo los graves y los no esperados, para mantener una alta confianza de la población en los programas de vacunaciones. La eficacia y algunos aspectos de la seguridad de las vacunas, en particular los efectos adversos a corto plazo o más frecuentes, se demuestran durante los ensayos clínicos controlados realizados antes de su comercialización. Después de registradas y comercializadas, se realizan estudios de farmacovigilancia que proporcionan información adicional sobre efectividad y riesgos ${ }^{2}$.

Un efecto adverso supuestamente atribuible a la vacunación o inmunización (ESAVI) es aquel evento clínico desfavorable que ocurre luego de la administración de una vacuna. Su aparición no implica que se trate de una verdadera reacción adversa por vacunas, sino que requiere de un adecuado análisis para establecer su imputabilidad ${ }^{3}$.

Desde el año 2010, el Ministerio de Salud Pública
(MSP) de Uruguay, lleva a cabo una vigilancia de los ESAVI a través de la Unidad de Farmacovigilancia y la Unidad de Inmunizaciones, basada en la notificación espontánea por parte de la población, principalmente profesionales de la salud. A pesar de ser un valioso insumo para la vigilancia, este sistema pasivo presenta debilidades universalmente reconocidas. Entre ellas, la sub-notificación y el requerimiento de que los ESAVI sean identificados como tales previo a su reporte ${ }^{4}$. Esto hace necesario comenzar a desarrollar actividades de vigilancia activa de ESAVI y así complementar y fortalecer al sistema. Los sistemas de vigilancia activa aportan una mejor caracterización de los ESAVI, sobre todo en cuanto a su frecuencia, relación causal y para estudiar poblaciones especiales o vacunas particulares. Sin embargo, requieren el diseño de estudios específicos y capacitación para poder realizarlos ${ }^{4}$.

Uruguay participa de la red mundial de vacunación segura de la Organización Panamericana de la Salud (OPS) y la Organización Mundial de la Salud (OMS) desde el año 2014, a través de la implementación de hospitales centinelas para la vigilancia de ESAVI. En nuestro país se seleccionó al Centro Hospitalario Pereira Rossell (CHPR) por cumplir con todos los requisitos necesarios para dicha función ${ }^{5-7}$. 
En este contexto se comenzaron a desarrollar estudios a nivel local con la finalidad de evaluar la factibilidad y calidad de la farmacovigilancia activa en dicho hospital. Se decidió caracterizar con mayor exactitud a las convulsiones febriles que son uno de los ESAVI graves más frecuentes reportados al Sistema Nacional de Farmacovigilancia y cuya potencial asociación con la vacunación ya es conocida ${ }^{8}$. Las convulsiones febriles se han visto asociadas al componente pertussis a células completas de las distintas vacunas combinadas que los contienen, como la vacuna difteria - tétanos - pertussis (DPT) ${ }^{9}$. Se caracterizan por ser, en general, convulsiones febriles simples, de carácter benigno, que no requieren tratamiento específico ni generan secuelas, pero generalmente requieren hospitalización u observación y provoca gran angustia en la familia. La frecuencia a nivel internacional es de 570/1.000.000 de dosis administradas 9.

En el Programa Nacional de Vacunaciones (PNV) de Uruguay el componente pertussis se administra como vacuna pentavalente a los 2, 4, 6 y 15 meses y como vacuna DPT a los 5 años. A esas mismas edades se administran otras vacunas que no han sido asociadas con convulsiones febriles: la vacuna antipoliomielítica inactivada a $\operatorname{los} 2$, 4, 6 y 15 meses, la antineumocócica 13 valente a los 2, 4 y 12 meses, la vacuna antihepatitis A a los 15 meses y la segunda dosis de la vacuna triple viral y antivaricela a los 5 años ${ }^{9,10}$.

El estudio de series de casos auto-controlados (SCCS, por sus siglas en inglés) es un diseño desarrollado para investigar posibles eventos adversos de inmunización, y es una alternativa a los estudios de casos y controles más clásicos o de cohorte ${ }^{11-13}$. En este diseño, sólo se incluyen los individuos que sufren el evento y cada individuo es su propio control. Los factores de confusión invariantes en el tiempo se eliminan y los períodos de exposición y de no exposición se definen dentro de cada individuo. El período de riesgo está predefinido y la incidencia relativa se calcula para ese período en comparación con otros tiempos que constituyen el período de control ${ }^{14,15}$.

El objetivo principal de este trabajo fue analizar la asociación de convulsiones febriles con la administración de la vacuna pentavalente en niños bajo 2 años de edad asistidos en el CHPR durante el año 2014.

\section{Material y Métodos}

Se realizó un estudio de series de casos auto-controlados. Se incluyeron niños procedentes de Montevideo entre 2 y 24 meses al momento de la consulta, con diagnóstico de convulsión febril al egreso del Departamento de Emergencia Pediátrica (DEP) del CHPR entre el 1 de enero y el 31 de diciembre de 2014.

Todas las dosis de vacuna pentavalente administra- das en el país durante el año 2014 corresponden a la marca Heberpenta-L (lotes 4AA1503/0, 4AA1504/0, 4AA0901/0, 4AA1202/0) que incluye el componente celular de Bordetella pertussis + toxoide diftérico + toxoide tetánico 'PRP de Haemophilus influenzae b + antígeno de superficie de hepatitis B.

\section{Criterios de inclusión}

Para este estudio se consideró convulsión febril aquella que ocurre en mayores de un mes de vida, sin antecedentes de convulsiones afebriles, que se presenta con temperatura axilar de $38^{\circ} \mathrm{C}$ o más y que no es consecuencia de infecciones en el sistema nervioso central ni de alteraciones metabólicas ${ }^{16}$. Para la selección de los casos se consideró aquellos pacientes que al egreso tenían como diagnóstico "Convulsiones/Crisis convulsivas". Este diagnóstico corresponde al código N07 de la Clasificación Internacional de Atención Primaria (CIAP) $2^{\circ}$ edición, que es el utilizado en el sistema de registro del DEP. Se seleccionaron los que cumplían con la definición de convulsión febril.

\section{Criterios de exclusión}

Se excluyeron los epilépticos y portadores de encefalopatía crónica.

De los niños incluidos del registro del DEP se analizaron sus historias clínicas y se registraron: nombre, cédula de identidad, fecha de nacimiento, fecha de primera consulta por el evento, fecha de consulta en el DEP y fecha de inicio del evento. Se consideró fecha de inicio del evento a la registrada en la historia clínica o, de no estarlo, a la primera fecha de consulta disponible para ese evento. Las readmisiones dentro de las primeras $72 \mathrm{~h}$ fueron consideradas como un evento único.

A partir de la cédula de identidad y el nombre de los casos incluidos se obtuvo el registro vacunal de cada niño disponible en el Sistema Nacional de Registro Nominal de la Comisión Honoraria para la Lucha Antituberculosa y Enfermedades Prevalentes (CHLA-EP). Para el análisis se consideró: fecha de administración de la vacuna pentavalente previa al evento y número relativo de dosis con su fecha correspondiente. Esta información fue recabada por integrantes de la Unidad de Inmunizaciones del MSP. Una vez combinados ambos registros se anonimizó la base de datos para el posterior análisis. La recolección de datos a partir de los casos y su vinculación con los registros de vacunación se realizó conforme a las normativas de protección de datos y de confidencialidad nacionales.

Se verificó si los casos fueron previamente notificados al Sistema Nacional de Farmacovigilancia del MSP y si, a los que les correspondía revacunación, volvieron a recibir las dosis correspondientes. Las fuentes para verificar estos datos fueron las bases de datos del Sistema Nacional 
Tabla 1. Distribución de eventos $(n=135)$ y denominadores para la estimación de la tasa, por períodos de tiempo*. Montevideo, Uruguay, 2014

\begin{tabular}{lrc}
\hline Período de tiempo & Eventos $\mathbf{n}(\%)$ & Persona-tiempo* $^{*}$ \\
\hline Período de no riesgo (> 14 días ) & $120(88,9 \%)$ & 35.650 días \\
\hline Período de riesgo ( 0 a 3 días) & $7(5,2 \%)$ & 512 días \\
\hline Período de lavado (4 a 14 días) & $8(5,9 \%)$ & 1.441 días \\
\hline Total & $135(100,0 \%)$ & \\
\hline
\end{tabular}

*Los valores de persona-tiempo se obtuvieron como la sumatoria de los tiempos de permanencia de cada paciente en cada período de tiempo.

Tabla 2. Distribución de eventos $(n=135)$ en períodos de riesgo y lavado, por dosis de vacuna pentavalente. Montevideo, Uruguay, 2014

\begin{tabular}{llllll}
\hline Período & \multicolumn{3}{c}{ Dosis } & \multicolumn{2}{c}{ Total } \\
\hline Riesgo (0 a 3 días post-vacunación) & $\mathbf{1}$ & $\mathbf{2}$ & $\mathbf{3}$ & $\mathbf{4}$ & \\
Lavado (4 a 14 días post-vacunación) & 1 & 0 & 4 & 3 & 7 \\
Total & 1 & 1 & 2 & 4 & 8 \\
\hline
\end{tabular}

Tabla 3. Riesgos relativos (RR) ajustados e intervalos de confianza del $95 \%$ (IC 95\%) por períodos de tiempo (períodos de riesgo y lavado) en relación con el período sin riesgo $(n=135)$. Montevideo, Uruguay, 2014

$\begin{array}{lccc}\text { Período } & \text { RR } & \text { IC 95\% } & \text { p } \\ \text { No riesgo (> 14 días post-vacunación) } & 1 & & \\ \text { Lavado (4 a 14 días post-vacunación) } & 1,71 & (0,75 ; 3,29) & >0,1 \\ \text { Riesgo (0 a 3 días post-vacunación) } & 4,14 & (1,73 ; 8,36) & <0,001\end{array}$

de Farmacovigilancia del MSP y el Sistema Nacional de Registro Nominal de vacunaciones de la CHLA-EP, respectivamente.

Para la descripción de las variables cualitativas se utilizaron frecuencias absolutas y relativas y para las variables cuantitativas media y rango.

Para la estimación de riesgo se utilizó la técnica de series de casos auto-controlados (SCCS) propuesto por Farrington ${ }^{12}$ y Whitaker et $\mathrm{al}^{17}$. Se determinó la "ventana temporal", entendida como la diferencia entre las fechas de inicio del evento y la de la vacunación. Se consideró periodo de riesgo si ocurría entre los 0 y 3 días postvacunación, imputando al evento como asociado a la vacunación. Al período entre 4 y 14 días se lo consideró como período de lavado, dado que la asociación con la vacunación no es concluyente. Se consideró el evento como no asociado con la vacunación si ocurría luego de los 14 días de administrada la vacuna. Al considerar no concluyente la asociación de eventos que ocurren dentro del período de lavado, se analizó este intervalo aparte del período de riesgo ${ }^{18}$.

\section{Análisis estadístico}

Se ajustó un modelo de regresión de Poisson condicional para los intervalos de riesgo y de lavado considerados ( 0 a 3 y 4 a 14 días post-vacunación) en comparación con el período considerado de no riesgo (más de 14 días postvacunación y hasta cumplir los 24 meses o hasta el fin del período de observación, el 31 de diciembre de 2014). No se consideraron cambios en el riesgo por intervalos de edad diferentes ni se analizaron por períodos de no riesgos más cortos. El procesamiento de datos se realizó utilizando el lenguaje de programación R 3.3.1 ${ }^{19}$; para el ajuste del modelo se utilizó la librería gnm. ${ }^{20}$. Se estimó el riesgo relativo para cada uno de los períodos (de riesgo y de lavado) comparados con el período de no riesgo. Se consideró un nivel de significación de 5\%.

\section{Aspectos éticos}

Se solicitó autorización a la Dirección del CHPR y MSP para llevar a cabo este trabajo y fue aprobado por el Comité de Ética del CHPR.

\section{Resultados}

En el período de estudio se incluyeron 135 convulsiones febriles, que ocurrieron en 114 niños, ninguno presentó readmisiones dentro de las primeras $72 \mathrm{~h} ; 16$ niños presentaron dos o más eventos.

De los 114 niños se obtuvo registro vacunal en 112 (98\%). No tenían dosis alguna administrada 2. La edad media al momento de la convulsión fue 15,9 meses (rango 3,8-24,3).

El total de eventos ocurridos en el período de riesgo fue $7(5,2 \%)$ y en el período de lavado 8 (5,9\%). Estos 15 $(11,1 \%)$ eventos correspondieron a 15 niños (Tabla 1$)$.

De los 15 niños, 3 tuvieron recurrencias luego de los primeros 14 días tras la vacunación (1 de ellos tuvo 3 eventos). Por protocolo no se indagó la causa de estos nuevos eventos.

En la Tabla 2 se muestra la relación de estos 15 casos con el número de dosis de vacuna pentavalente administrada. La mayor frecuencia de convulsiones se observó tras la $3^{\circ}$ y $4^{\circ}$ dosis.

En la Tabla 3 se muestra la estimación del riesgo relativo ajustados para cada uno de los intervalos comparados con el período de no riesgo.

Según los datos del Sistema Nacional de Farmacovigilancia, durante el año 2014 no se recibió notificación alguna de convulsiones febriles. 
Le correspondía continuar con el esquema de inmunización con vacuna pentavalente a 8 de los 15 niños ( 4 del período de riesgo y 4 del período de lavado).

Todos los que se encontraban en período de riesgo la recibieron y 3 de los 4 que se encontraban en período de lavado. El niño que no recibió la vacuna pentavalente no recibió otras inmunizaciones.

Por protocolo no se evaluó la reiteración de convulsiones con estas dosis.

\section{Discusión}

Este fue el primer trabajo a nivel nacional realizado en el CHPR como hospital centinela para la vigilancia de la seguridad de vacunas. Fue llevado a cabo por un equipo interdisciplinario, aplicando el análisis de series de casos auto-controlados. Esta metodología para el desarrollo de estudios de farmacovigilancia activa es alternativo a los estudios de caso-control y cohortes. Fue desarrollado específicamente para investigar la asociación entre la vacunación y la aparición de eventos adversos. Varios trabajos de farmacovigilancia y farmacoepidemiología han comenzado a utilizar esta metodología $a^{5-7,21-25}$. Tiene como ventajas que utiliza sólo casos (estima una incidencia relativa), no necesita denominadores poblacionales y permite controlar las variables de confusión ${ }^{11}$. Sin embargo, este tipo de análisis requiere asumir algunos supuestos necesarios para la correcta interpretación de los datos que constituyen limitantes del diseño. En primer lugar, que todos los eventos ocurridos durante el intervalo de riesgo son atribuibles a la exposición, es decir a la vacuna pentavalente. En segundo lugar, la recurrencia de los eventos debe ser independiente o eventos raros no recurrentes: la aparición de un evento no aumenta la posibilidad de un segundo evento. Tercero, la ocurrencia de un evento no debe censurar o modificar el período de observación. Finalmente, en cada período definido, la probabilidad de ocurrencia debe ser constante en el tiempo ${ }^{11}$. Datos en la bibliografía consultada indican que la probabilidad de convulsiones febriles por componente pertussis es mayor en los primeros tres días post-vacunación ${ }^{26}$. En este caso se consideraron las convulsiones febriles como eventos independientes cuando ocurrían separadas por intervalos mayores a $72 \mathrm{~h}$. Las convulsiones febriles no son letales ni contraindican la posterior exposición como lo señalan las recomendaciones del $\mathrm{MSP}^{27}$.

Otro supuesto es que es necesario asegurar que el investigador sea capaz de captar todos los casos que ocurren del evento de interés en la población objetivo. Por este motivo, en este estudio se limitó el área geográfica de procedencia de los pacientes incluidos exclusivamente a Montevideo, capital del país. Todos los pacientes del sub-sector público de atención en salud que tengan una convulsión febril son finalmente asistidos en el DEP del CHPR donde se realizó el estudio, ya que existe una pauta nacional que establece que estos niños sean observados por $12 \mathrm{~h}$ en un área de emergencia siendo el DEP el único que reúne los requisitos para este fin.

En este estudio, 5,2\% de las convulsiones febriles ocurrieron durante el período de riesgo, lo que corresponde a una tasa de 4,99 casos por persona-año. La tasa para el período sin riesgo fue de 1,23 casos cada una persona-año. Para estimar la frecuencia de ESAVI, los informes publicados usan las tasas, relacionando el número de ESAVI registrados con las dosis administradas o distribuidas en una población dada ${ }^{28}$. Si se toma como denominador las dosis administradas en Montevideo en el período de estudio, se estimaría una tasa de 19 convulsiones febriles por cada millón de dosis administradas, un valor muy inferior a la tasa internacional publicada de 570 casos por cada millón de dosis administradas 9 . Sin embargo, nuestro valor está subestimado, ya que se desconoce el número de dosis administradas en la población atendida por el hospital centinela. Coincidiendo con la literatura médica disponible, los primeros tres días después de la vacunación mostraron un mayor riesgo de sufrir una convulsión ${ }^{9,18}$. No se pudo demostrar un aumento significativo en el riesgo durante el período de lavado, en comparación con el período sin riesgo. Si bien se reconoce que el riesgo es mayor para la tercera y cuarta dosis ${ }^{18}$, no se logró estimar el riesgo para cada dosis, dado el pequeño número de sujetos incluidos.

A pesar de la ocurrencia de casos que se registraron en este trabajo, ninguno de los eventos fue notificado al Sistema Nacional de Farmacovigilancia. La sub-notificación puede estar relacionada con la falta de conocimiento de los profesionales clínicos sobre el sistema de notificación o falta de sospecha de la posible asociación entre las convulsiones y la vacunación ${ }^{29}$. Los médicos clínicos deben conocer el evento y conocer los períodos de riesgo para así interpretar de forma adecuada y notificar al MSP. Sospechar la ocurrencia de un ESAVI y notificarlo no implica aseverar que el mismo sea consecuencia de la vacunación. Es necesario considerar todas las posibilidades etiológicas y recordar que la caracterización como ESAVI es un diagnóstico por exclusión. A pesar de esto, es necesario incorporar al razonamiento clínico, frente a un niño con convulsiones febriles, la noción de riesgo potencialmente asociada con la vacunación ${ }^{30}$.

Es importante señalar que fue posible implementar en el CHPR la vigilancia activa de estos eventos, permitiendo su mejor caracterización. Esto representa una fortaleza del hospital como centro centinela, parte de una red colaborativa internacional que brinde respuesta al conocimiento de la seguridad de nuevas vacunas o eventos menos conocidos? 


\section{Conclusiones}

Este trabajo permitió establecer una línea de base en el país para el riesgo de convulsiones febriles asociadas con la vacunación pentavalente, al utilizar, por primera vez en el país, una metodología diseñada específicamente para este objetivo. No obstante, la atribución de causalidad requiere de un análisis que incluya otros elementos. También permitió demostrar la relevancia de CHPR como centro centinela para la vigilancia activa de ESAVI.

\section{Resumen}

Introducción: Las convulsiones febriles son eventos supuestamente atribuidos a la vacunación e inmunización (ESAVI) frecuentemente asociados a vacuna difteria - tétanos- pertussis a células completas. Objetivo: Analizar la asociación de convulsiones febriles con la administración de la vacuna pentavalente en niños bajo 2 años de edad asistidos en el Centro Hospitalario Pereira Rossell durante el año 2014. Material y Métodos: Estudio de series de casos auto-controlados. Se incluyeron niños procedentes de Montevideo con 2 a 24 meses de edad con diagnóstico de convulsión febril. Se estimó el riesgo relativo (RR) de los períodos de riesgo ( 0 a 3 días) y de lavado ( 4 a 14 días), en comparación con el período de no riesgo (más de 14 días). Resultados: Se registraron 135 convulsiones febriles, que ocurrieron en 114 niños; 16 niños presentaron dos o más episodios. El total de eventos ocurridos en el período de riesgo fueron $7(5,2 \%)$ y $8(5,9 \%)$ en el período de lavado. El período de riesgo mostró un RR significativo de 4,14 (IC 95\%: 1,73; 8,36). Conclusiones: Este trabajo permitió establecer una línea de base nacional sobre el riesgo de convulsiones febriles asociadas con la vacunación pentavalente al utilizar por primera vez en el país una metodología específica para tal fin.

\section{Referencias bibliográficas}

1.- Tregnaghi M W. Ética de la inmunización. En: Tregnaghi MW. Manual de vacunas de Latinoamérica. $3^{\circ}$ ed. Asociación Panamericana de Infectología; 2005. pp: 551-7. http:// infectologiapediatrica.com/attachments/ manual_vacunas.pdf.

2.- Organización Panamericana de la Salud. Vacunación Segura: cómo enfrentar los eventos supuestamente atribuidos a la vacunación o inmunización. Washington D.C: División de Vacunas e Inmunización OPS; 2002. http://www.who.int/immunization_safety/ publications/aefi/en/vacunacion_segura_S.pdf.

3.- Tregnaghi M W. Reacciones adversas de las vacunas y farmacovigilancia. En: Tregnaghi MW. Manual de vacunas de Latinoamérica. $3^{\circ}$ edición. Asociación Panamericana de Infectología; 2005. pp 511-26. http:// infectologiapediatrica.com/attachments/ manual_vacunas.pdf.

4.- Red Panamericana de Armonización de la Reglamentación Farmacéutica. Buenas prácticas de farmacovigilancia para las Américas. Disponible en: http://apps.who.int/ medicinedocs/documents/s18625es/s18625es. pdf.

5.- Pérez-Vilar S, Weibel D, Sturkenboom M, Black S, Maure C, Castro J L, et al. Enhancing global vaccine pharmacovigilance: Proofof-concept study on aseptic meningitis and immune thrombocytopenic purpura following measles-mumps containing vaccination. Vaccine, 2018; 36 (3): 347-4. doi: 10.1016/j. vaccine.2017.05.012.

6.- Maure C, Elango V, Black S, Pérez-Vilar
S, Castro J L, Bravo-Alcántara P, et al. Operational lessons learned in conducting a multi-country collaboration for vaccine safety signal verification and hypothesis testing: The Global Vaccine Safety Multi Country Collaboration initiative. Vaccine 2018; 36 (3): 355-62. doi: 10.1016/j. vaccine.2017.07.085.

7.- Bravo P, Pérez S, Molina H, Sturkenboom M, Black S, Maure C, et al. Building capacity for active surveillance of vaccine adverse events in the Americas: A hospital-based multicountry network. Vaccine 2018; 36 (3): 363-70. doi: 10.1016/j.vaccine.2017.04.069.

8.- Informe Sistema Notificación de ESAVI 2013. Inmunizaciones, División EpidemiologíaUnidad de Farmacovigilancia, División Evaluación Sanitaria. http://www.msp.gub. uy/sites/default/files/archivos_adjuntos/ INFORMEESAVI2013_0.pdf.

9.- Tregnaghi M W. Vacunación contra la Difteria, Tétanos, y Tos Ferina (DPT). En: Tregnaghi MW. Manual de vacunas de Latinoamérica. $3^{\circ}$ ed. Asociación Panamericana de Infectología; 2005. pp: 75 - 99. http://infectologiapediatrica. com/attachments/manual_vacunas.pdf.

10.- Uruguay. Ministerio de Salud. Programa Ampliado de Inmunizaciones. http://www. msp.gub.uy/publicaci\% $\%$ C3\%B3n/programanacional-de-vacunaciones

11.- Whitaker H, Hocine M, Farrington P. The methodology of self-controlled case series studies. Stat Methods Med Res 2009; 18 (1): 7-26. doi: 10.1177/0962280208092342.

12.- Farrington C. Relative incidence estimation from case series for vaccine safety evaluation. Biometrics 1995; 51 (1): 228-35. PMID:
7766778

13.- Whitaker H, Paddy Farrington C, Spiessens $\mathrm{B}$, Musonda P. Tutorial in biostatistics: the self-controlled case series method. Statistics in Medicine 2006; 25 (10): 1768-97. doi: 10.1002/ sim. 2302 .

14.- Weldeslassie $Y$, Whitaker $H$, Farrington C. Use of the self-controlled case-series method in vaccine safety studies: review and recommendations for best practice. Epidemiol Infect 2011; 139(12): 1805-17. doi: 10.1017/ S0950268811001531.

15.- Petersen I, Douglas I, Whitaker H. Selfcontrolled case series methods: an alternative to standard epidemiological study designs. Br Med J 2016; 354: i4515. doi: https://doi. org/10.1136/bmj.i4515.

16.- Guidelines for epidemiologic studies on epilepsy. Commission on Epidemiology and Prognosis, International League Against Epilepsy. Epilepsia. 1993; 34: 592-6. doi: 10.1111/j.1528-1157.1993.tb00433.x.

17.- Whitaker, H, Farrington C, Spiessens B, Musonda P. Tutorial in Biostatistics: the selfcontrolled case series method. Statistics in Medicine 2005; 0: 1-31. http://statistics.open. ac.uk/sces/sccs.pdf.

18.- Farrington P, Pugh S, Colville A, Flower A, Nash J, Morgan-Capner P, et al. A new method for active surveillance of adverse events from diphtheria/tetanus/pertussis and measles/ mumps/rubella vaccines. Lancet 1995; 345 : 567-9. doi: 10.1016/s0140-6736(95)90471-9.

19.- Core R R. A language and environment for statistical computing. R Foundation for Statistical Computing, Vienna, Austria. 2016. https://www.R-project.org/. 
20.- Turner H, Firth D. Generalized nonlinear models in R: An overview of the gnm package. (R package version 1.0-8).2015. http:// CRAN.R-project.org/package $=$ gnm.

21.- Asghar Z, Coupland C, Siriwardena N. Influenza vaccination and risk of stroke: Self-controlled case-series study. Vaccine. 2015; 33 (41): 5458-63. doi: 10.1016/j. vaccine.2015.08.013.

22.- Cohet C, Haguinet F, Dos Santos G, Webb D, Logie J, Ferreira G, et al. Effect of the adjuvanted (AS03) A/H1N1 2009 pandemic influenza vaccine on the risk of rejection in solid organ transplant recipients in England: a self-controlled case series. $\mathrm{Br}$ Med J Open 2016;6(1): 1-10. doi: 10.1136/ bmjopen-2015-009264.

23.- Crawford N W, Cheng A, Andrews N, Charles PG, Clothier H J, Day B, et al. Guillain-Barré syndrome following pandemic (H1N1) 2009 influenza A immunisation in Victoria: a selfcontrolled case series. Med J Aust 2012; 197 (10): 574-8. doi: 10.5694/mja12.10534.

24.- Yung C F, Chan S P, Soh S, Tan A, Thoon K C. Intussusception and monovalent rotavirus vaccination in Singapore: Self-controlled case series and risk-benefit study. J Pediatr. 2015; 167 (1): 163-8. doi: 10.1016/j. jpeds.2015.03.038.

25.- Pérez-Vilar S, Díez-Domingo J, Puig-Barberà J, Gil-Prieto R, Romio S. Intussusception following rotavirus vaccination in the Valencia Region, Spain.Hum Vaccin. 2015; 11 (7): 184852. doi: 10.1080/21645515.2015.1049787.

26.- World Health Organization. Information sheet observes rate of vaccines Diphteria, Pertussis, Tetanus Vaccines. 2014. http://www.who.int/ vaccine_safety/initiative/tools/DTP_vaccine_ rates_information_sheet.pdf.

27.- Picón T, Speranza N, Varela A, Zunino C.
Vacunación en situaciones especiales. $2^{\circ}$ ed. Montevideo: MSP; 2015. https://www.gub. uy/ministerio-salud-publica/sites/ministeriosalud-publica/files/documentos/publicaciones/ Vacunacion $\% 20$ en $\% 20$ situaciones $\% 20$ especiales.pdf.

28.- World Health Organization. Global manual on surveillance of adverse events following immunization. 2014. https://www.who.int/ vaccine_safety/publications/Global_Manual_ revised_12102015.pdf?ua $=1$.

29.- Informe Sistema Notificación de ESAVI 2014. Inmunizaciones, División EpidemiologíaUnidad de Farmacovigilancia, División Evaluación Sanitaria. http://www.msp.gub.uy/ sites/default/files/archivos_adjuntos/2014.pdf.

30.- Capellà D, Laporte J R. La notificación espontánea de reacciones adversas a medicamentos. http://www.icf.uab.es/pem/docs/ cap8.pdf. 